\title{
Erratum
}

\section{The old open clusters Berkeley 36, Berkeley 73 and Biurakan 13 (Berkeley 34)^}

\author{
S. Ortolani ${ }^{1}$, E. Bica ${ }^{2}$, B. Barbuy ${ }^{3}$, and M. Zoccali ${ }^{4}$ \\ 1 Università di Padova, Dipartimento di Astronomia, Vicolo dell'Osservatorio 2, 35122 Padova, Italy \\ e-mail: ortolani@pd.astro.it \\ 2 Universidade Federal do Rio Grande do Sul, Departamento de Astronomia, CP 15051, Porto Alegre 91501-970, Brazil \\ e-mail: bica@if.ufrgs.br \\ 3 Universidade de São Paulo, IAG, Rua do Matão 1226, Cidade Universitária, São Paulo 05508-900, Brazil \\ e-mail: barbuy@astro.iag.usp.br \\ 4 Universidad Catolica de Chile, Department of Astronomy \& Astrophysics, Casilla 306, Santiago 22, Chile \\ e-mail: mzoccali@astro.puc.cl
}

A\&A, 429, 607-612 (2005), DOI: 1021051/0004-6361:20041458

\begin{abstract}
The coordinates of Berkeley 73 typed in the paper are wrong. The correct ones are $\mathrm{J} 2000 \alpha=06^{\mathrm{h}} 22^{\mathrm{m}} 05^{\mathrm{s}}, \delta=$ $-06^{\circ} 18^{\prime} 59^{\prime \prime}\left(l=215.26^{\circ}, b=-9.39^{\circ}\right)$. The coordinates in the Galaxy were revised to $X=-13.5 \mathrm{kpc}, Y=-3.9 \mathrm{kpc}$, $Z=-1.1 \mathrm{kpc}$, and the distance from the Galactic center $R_{\mathrm{GC}}=14.1 \mathrm{kpc}$. We confirm that the cluster images and colourmagnitude diagrams shown in the paper are indeed from Berkeley 73.
\end{abstract}

Key words. Galaxy: open clusters and associations: individual: Berkeley 36, Berkeley 73, Biurakan 13 - HR diagram errata, addenda 